\title{
Right Atrial Thrombosis in Antiphospholipid Syndrome with Secondary Immune Thrombocytopenia
}

\author{
Minna Voigtlaender ${ }^{1}$ Lenard Conradi ${ }^{2}$ Andrea Hinsch ${ }^{3} \quad$ Florian Langer $^{1}$ \\ ${ }^{1}$ Universitätsklinikum Hamburg-Eppendorf, II. Medizinische Klinik und \\ Poliklinik, Onkologisches Zentrum, Hamburg, Germany \\ 2 Universitätsklinikum Hamburg-Eppendorf, Klinik und Poliklinik für \\ Herz- und Gefäßchirurgie, Universitäres Herzzentrum, Hamburg, \\ Germany \\ 3 Universitätsklinikum Hamburg-Eppendorf, Institut für Pathologie, \\ Hamburg, Germany \\ Thorac Cardiovasc Surg Rep 2015;4:40-43. \\ Address for correspondence Florian Langer, MD, Universitätsklinikum \\ Hamburg-Eppendorf, II. Medizinische Klinik und Poliklinik, \\ Onkologisches Zentrum, Hamburg, Germany \\ (e-mail: f.langer@uke.de).
}

Background Antiphospholipid syndrome (APS) is an acquired thrombophilia that can be associated with decreased platelet counts.

Case A 67-year-old woman presented with thrombocytopenia and a symptomatic right atrial mass suspicious of cardiac myxoma. Prolongation of the activated partial thromboplastin time (aPTT) was caused by a strong lupus anticoagulant, and bone marrow cytology was consistent with accelerated platelet clearance. The patient underwent uneventful resection of the atrial tumor, which turned out to be a calcified fibrin-rich thrombus. Definitive APS was diagnosed and long-term anticoagulation recommended.

Conclusion When evaluating patients with right atrial masses, findings of thrombocytopenia and/or aPTT prolongation should raise the suspicion of APS-associated thrombosis.

\section{Introduction}

Antiphospholipid syndrome (APS) is an autoimmune disorder characterized by vascular thrombosis and/or pregnancy morbidity in the presence of antiphospholipid antibodies (APAs), which are usually detected as cardiolipin or $\beta_{2}$-glycoprotein I $\left(\beta_{2}\right.$-GPI) antibodies or as lupus anticoagulant (LA). ${ }^{1}$

Although not required to establish the diagnosis of definitive APS according to the revised classification criteria, thrombocytopenia is a quite common laboratory phenomenon in patients with APS with a reported prevalence of 20 to $40 \%{ }^{2}$ The pathophysiology of APS-associated thrombocytopenia is thought to be similar to that of immune thrombocytopenia (ITP), although it remains a matter of debate whether APAs themselves or other antibodies directed against platelet membrane glycoproteins are responsible for accelerated platelet clearance by the reticuloendothelial system. ${ }^{3}$

Here, we describe the unusual case of an elderly woman presenting with symptomatic tricuspid valve dysfunction because of a right atrial mass. Although initial findings of thrombocytopenia and a prolonged activated partial thromboplastin time (aPTT) were indicative of a bleeding rather than a prothrombotic disorder, the cardiac mass turned out to be a calcified fibrin-rich thrombus, which, in combination with detection of a persistent LA, established the diagnosis of definitive APS with secondary ITP.

\section{Case Description}

A 67-year-old woman presented to another hospital with a 5week history of palpitations, angina pectoris, and progressive
License terms

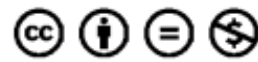

received

December 22, 2014 accepted after revision March 18, 2015

published online

April 20, 2015
DOI http://dx.doi.org/ 10.1055/s-0035-1549841. ISSN 2194-7635.

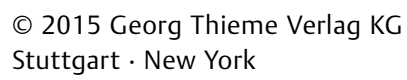


shortness of breath. She had never smoked, did not take any medication, and had no history of symptomatic or asymptomatic arterial or venous thromboembolism. In addition, there was no history of pregnancy complications. The patient had been pregnant once and had delivered a healthy child. Her family history was unremarkable. On physical examination, there was pitting edema, but no other finding of cardiac or pulmonary disease. Laboratory work-up revealed isolated moderate thrombocytopenia with a peripheral platelet count of $57 \times 10^{9} / \mathrm{L}$ (normal, $150-350 \times 10^{9} / \mathrm{L}$ ) and a prolonged aPTT of 45 seconds (normal, 26-37 seconds). The prothrombin time and fibrinogen level were normal as were kidney and liver function tests.

Transthoracic and transesophageal echocardiography showed an echogenic, mobile mass of $2.5 \times 1.5 \mathrm{~cm}$ in the right atrium, consistent with a primary cardiac tumor (-Fig. 1). The tumor had close contact to the septal leaflet of the tricuspid valve and collapsed into the right ventricle during systolic contraction, resulting in moderate tricuspid valve regurgitation. There were no other functional or structural heart valve abnormalities. Left ventricular function was normal with an estimated ejection fraction of $61 \%$. Specifically, there were no regional wall motion abnormalities suggestive of myocardial microangiopathy. Furthermore, significant atherosclerosis or other stenotic lesions of epicardial arteries were excluded by coronary angiography, which also showed no evidence for neovascularization of the cardiac tumor. Pulmonary embolism was excluded by computed tomography (CT) scanning of the chest. On the basis of the tentative diagnosis of right atrial myxoma with concomitant thrombocytopenia of unknown etiology, the patient was referred to our hospital for further diagnostic work-up and surgical treatment.

On referral, the patient denied a history of excessive bleeding. According to medical records, thrombocytopenia of 60 to $70 \times 10^{9} / \mathrm{L}$ had first been documented 4 weeks earlier. At that time and during the preceding 6 months, the patient had not been exposed to heparin. EDTA-induced

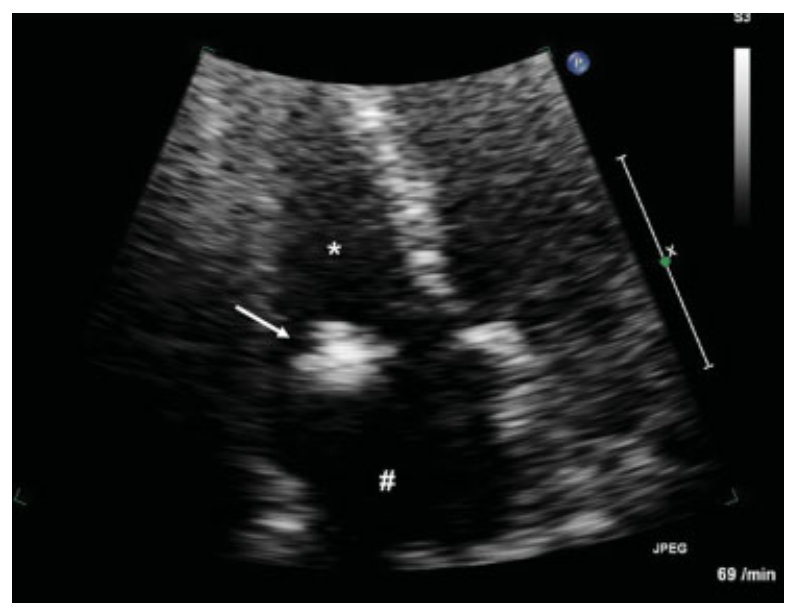

Fig. 1 Transthoracic echocardiography showing an echogenic, mobile mass $(\rightarrow)$ in the right atrium (\#) with close contact to the tricuspid valve; asterisk $\left(^{*}\right)$ indicates right ventricle. pseudothrombocytopenia was excluded by reproducing the decreased platelet count in citrate anticoagulated whole blood and by absence of platelet aggregates on peripheral blood smear. Serologic tests for HIV and hepatitis were negative. CT scanning of the chest and abdomen did not reveal any other tumor manifestations. Bone marrow cytology showed normal to slightly increased numbers of megakaryocytes, consistent with accelerated platelet clearance (-Fig. 2). There were no abnormal morphological or immunophenotypic findings indicative of a dysplastic or a myeloor lymphoproliferative disorder, suggesting ITP as the most likely cause of thrombocytopenia. Further work-up of the repeatedly prolonged aPTT revealed a strong LA in both an aPTT and a dilute Russel viper venom time (dRVVT)-based assay. Immunologic testing for cardiolipin and $\beta_{2}$-GPI antibodies was negative.

To minimize the risk of surgical bleeding, the patient received intravenous prednisolone at a dose of $150 \mathrm{mg}$ per day. Within 3 days of treatment, the platelet count increased to $79 \times 10^{9} / \mathrm{L}$, and uneventful open heart surgery with resection of the right atrial mass during cardiopulmonary bypass was performed without prophylactic platelet transfusion. The affected tricuspid valve was repaired by ring annuloplasty. On histological examination, the cardiac tumor turned out to be a calcified fibrin-rich thrombus (-Fig. 3 ).

The postoperative course was uneventful. Echocardiography showed normal ventricular function and no evidence of persisting tricuspid valve regurgitation. Treatment with prednisolone was stopped after 5 days. At that time, the platelet count had increased to $100 \times 10^{9} / \mathrm{L}$. Oral anticoagulation with phenprocoumon, a vitamin $\mathrm{K}$ antagonist (VKA), was initiated, and daily doses were titrated to maintain an international normalized ratio (INR) of 2.0 to 3.0. The patient was discharged 5 days after surgery. At 3 months of follow-up, the patient was symptom-free with no evidence for recurrent cardiac thrombosis. Laboratory work-up showed a normal platelet count of $320 \times 10^{9} / \mathrm{L}$ and persistence of the $\mathrm{LA}$,

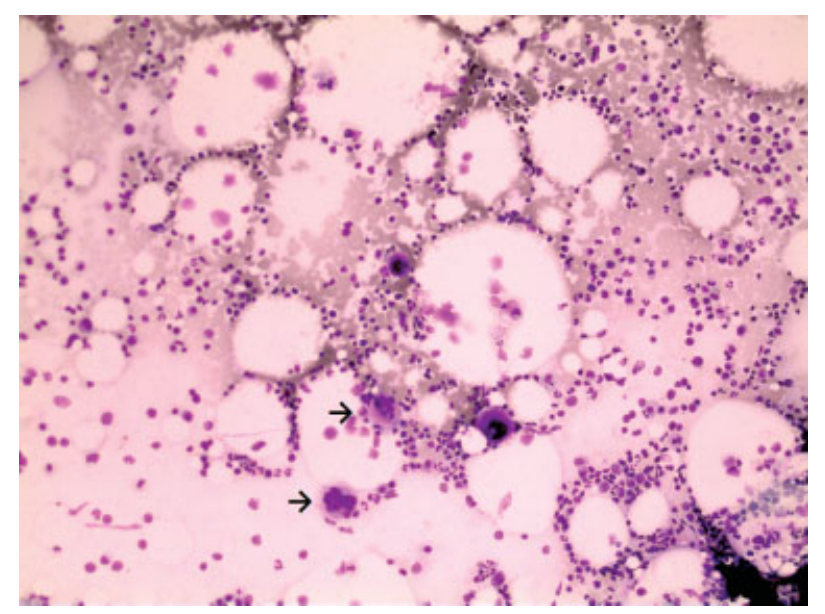

Fig. 2 Bone marrow cytology (May-Grünwald-Giemsa stain) showed normal-to-increased numbers of megakaryocytes $(\rightarrow)$, consistent with a diagnosis of immune thrombocytopenia. Picture was taken through a $\times 40$ objective. 


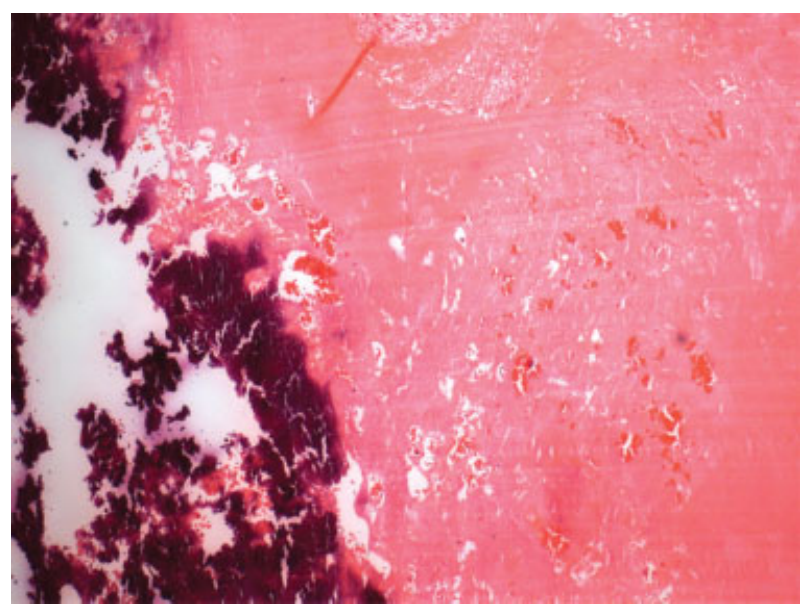

Fig. 3 Histological examination of the right atrial mass revealed a calcified fibrin-rich thrombus (hematoxylin and eosin stain, $\times 5$ ).

establishing the diagnosis of definitive APS. Long-term oral anticoagulation was recommended.

\section{Discussion}

In our patient, the calcified thrombus and the thrombocytopenia were likely caused by APS with secondary ITP. The patient fulfilled the revised classification criteria for definitive APS based on the following findings: presence of a histologically confirmed thrombosis and detection of a persistent LA. ${ }^{1}$ The diagnosis of ITP was suggested by isolated thrombocytopenia of $<100 \times 10^{9} / \mathrm{L}$, an unremarkable medical history, and compatible findings on peripheral blood smear and bone marrow cytology. Because decreased platelet counts had not become apparent before the onset of cardiac symptoms, it is reasonable to speculate that thrombocytopenia was an epiphenomenon accompanying APS rather than a distinct autoimmune disorder. Conversely, approximately $40 \%$ of patients with chronic ITP are tested positive for APAs, which may elevate the risk of developing future APS. ${ }^{3}$ Either way, clinicians should maintain a low threshold of suspicion for APS-associated thrombosis not despite, but because of otherwise unexplained thrombocytopenia, particularly when associated with a prolonged aPTT. In our patient, aPTT prolongation was caused by a strong LA, whereas tests for cardiolipin and $\beta_{2}$-GPI antibodies were negative. While a triple positive antibody profile may confer a high risk of thrombosis in the general population with APS, single LA positivity is an important determinant of the thromboembolic risk in patients with ITP. ${ }^{3}$

Immunosuppressive therapy appears to be efficacious in patients with APS-associated thrombocytopenia, suggesting that these patients can be treated similarly to those with chronic ITP. ${ }^{2}$ Consistently, a 5-day course of intravenous prednisolone (150 mg/day) significantly increased platelet counts in our patient. Although a response to corticosteroids usually occurs not earlier than 4 to 14 days after treatment initiation, administration of high doses of intravenous immunoglobulins may evoke a more rapid increase in platelet counts (i.e., within 24 hours) and should thus be considered in emergency situations such as acute bleeding or nondeferrable surgery. ${ }^{4}$ In our patient, a rather short-term course of prednisolone induced a long-lasting effect with platelet counts within the reference range at 3 months of follow-up. However, oral anticoagulation may have contributed to the sustained resolution of thrombocytopenia by inhibiting thrombin-mediated platelet activation. ${ }^{5}$

So far, only few cases of APS-associated right atrial thrombosis have been reported. ${ }^{6-8}$ In our patient, a clear distinction between right atrial myxoma as the most common primary tumor of the heart and cardiac thrombosis was not possible based on preoperative echocardiographic and angiographic findings. Although contrast-enhanced echocardiography and cardiac MRI may provide valuable additional information in this context, we favored surgical resection over further imaging studies. This decision was mainly based on the severely affected tricuspid valve and both the size and mobility of the intracardiac mass. Other cardiac manifestations of the APS include aseptic valve lesions, particularly of the mitral and aortic valves, coronary artery disease, and ventricular dysfunction because of the myocardial microthrombosis. ${ }^{1}$ However, neither echocardiographic nor angiographic findings were indicative of significant coronary macro- or microangiopathy in our patient.

In our patient, anticoagulation was indicated for two reasons. First, tricuspid valve repair by ring annuloplasty necessitated VKA treatment for 3 months. Second, longterm anticoagulation is standard treatment for the prevention of recurrent thrombosis in definitive APS. ${ }^{9}$ In this regard, the use of VKA can pose practical difficulties in some patients with APS, because, dependent on the prothrombin time reagent, INR values may be significantly influenced by a strong LA. Although target-specific oral anticoagulants (TSOACs) such as the factor Xa inhibitors rivaroxaban and apixaban or the thrombin inhibitor dabigatran do not require routine monitoring of their anticoagulant effects in clinical practice, recent case reports on patients with APS experiencing recurrent thromboembolic events while on therapy with TSOACs have questioned their usefulness as safe and efficacious alternatives in this highly prothrombotic disorder. ${ }^{10}$ Larger studies are thus needed to further define the role of TSOACs in patients with definitive APS.

Immunosuppressive therapy was commenced to reduce the risk of surgical bleeding, but such treatment may also be indicated in severely thrombocytopenic patients with APS to facilitate systemic anticoagulation. In contrast, immunosuppressive therapy is not routinely recommended in patients with APS without accompanying ITP, although several immunomodulatory agents are currently being investigated in clinical or experimental studies. ${ }^{11}$

In conclusion, in patients presenting with right atrial masses, concomitant findings of thrombocytopenia and/or aPTT prolongation should raise the suspicion of APS-associated cardiac thrombosis. Further testing for APAs is warranted, because positive results will likely affect both shortand long-term patient management. 


\section{References}

1 Miyakis S, Lockshin MD, Atsumi T, et al. International consensus statement on an update of the classification criteria for definite antiphospholipid syndrome (APS). J Thromb Haemost 2006;4(2): 295-306

2 Galli M, Finazzi G, Barbui T. Thrombocytopenia in the antiphospholipid syndrome. Br J Haematol 1996;93(1):1-5

3 Diz-Küçükkaya R, Hacihanefioğlu A, Yenerel M, et al. Antiphospholipid antibodies and antiphospholipid syndrome in patients presenting with immune thrombocytopenic purpura: a prospective cohort study. Blood 2001;98(6):1760-1764

4 Rodeghiero F, Stasi R, Gernsheimer T, et al. Standardization of terminology, definitions and outcome criteria in immune thrombocytopenic purpura of adults and children: report from an international working group. Blood 2009;113(11):2386-2393

5 Wisbey HL, Klestov AC. Thrombocytopenia corrected by warfarin in antiphospholipid syndrome. J Rheumatol 1996;23(4):769-771

6 Cianciulli TF, Saccheri MC, Redruello HJ, et al. Right atrial thrombus mimicking myxoma with pulmonary embolism in a patient with systemic lupus erythematosus and secondary antiphospholipid syndrome. Tex Heart Inst J 2008;35(4):454-457

7 Basso C, Bottio T, Rubino M, et al. Antiphospholipid syndrome and right atrial mass. J Thorac Cardiovasc Surg 2005;130(5): 1462-1463

8 Lim E, Wicks I, Roberts LJ. Intracardiac thrombosis complicating antiphospholipid antibody syndrome. Intern Med J 2004;34(3): 135-137

9 Ruiz-Irastorza G, Cuadrado MJ, Ruiz-Arruza I, et al. Evidence-based recommendations for the prevention and long-term management of thrombosis in antiphospholipid antibody-positive patients: report of a task force at the 13th International Congress on antiphospholipid antibodies. Lupus 2011;20(2):206-218

10 Win K, Rodgers GM. New oral anticoagulants may not be effective to prevent venous thromboembolism in patients with antiphospholipid syndrome. Am J Hematol 2014;89(10):1017

11 Pierangeli SS, Erkan D. Antiphospholipid syndrome treatment beyond anticoagulation: are we there yet? Lupus 2010;19(4): 475-485 